\title{
Maturity assessment of tomato fruit based on electrical impedance spectroscopy
}

\author{
J inyang Li*, Yao Xu, Wenjing Zhu, Xinhua Wei, Hongwei Sun \\ (Key Laboratory of Modern Agricultural Equipment and Technology, Ministry of Education of the People's Republic of China; \\ Institute of Agricultural Engineering, Jiangsu University, Zhenjiang 212013, China)
}

\begin{abstract}
Ripening is important to tomato quality, taste and nutrition. In this study, the maturity of plant-fruit was on-line investigated based on electrical impedance spectroscopy (EIS). The electrodes with sensing unit for contact force between samples and electrodes were designed. After fruits turn into green-white, impedance measurements were conducted on the fruit samples at various ripening stages in the range of $1 \mathrm{~Hz}$ to $1 \mathrm{MHz}$. The optimal frequencies $(100 \mathrm{~Hz}, 1 \mathrm{kHz}$ and $1 \mathrm{MHz})$ for maturity assessment were selected and five electrical impedance parameters at three sensitive frequencies were determined. The equivalent circuit model with CPE was developed and the model performance was evaluated. The soluble solid content and $\mathrm{pH}$ of fruit were determined and analyzed to explain the variations in EIS parameters sufficiently. Results showed that the impedance, phase angle, resistance, reactance and capacitance increased with the progression of maturity. The selected impedance parameters could be used to classify tomato samples into immature class or mature class with the accuracy of $88.3 \%$. Impedance analysis for different samples from the same branch demonstrated that the ripening stage of all other samples could be predicted and assessed by the impedance spectroscopy from one sample.
\end{abstract}

Keywords: tomato fruit, maturity, discriminate analysis, electrical impedance spectroscopy DOI: $10.25165 /$ j.ijabe.20191204.4664

Citation: Li J Y, Xu Y, Zhu W J, Wei X H, Sun H W. Maturity assessment of tomato fruit based on electrical impedance spectroscopy. Int J Agric \& Biol Eng, 2019; 12(4): 154-161.

\section{Introduction}

Fruit quality is closely related to the fruit maturity degree ${ }^{[1]}$. The appropriate ripening of fruits offers better taste with the desired nutrients. However, both the unripe and overripe are not satisfactory. Hence, in order to determine an optimum ripening state which all the essential and important nutrients can be supplied, it is essential to study the ripening process of fruit. Furthermore, a great deal of information about the physiological and biochemical changes emerging in the fruits can be obtained by monitoring the ripening process ${ }^{[2]}$. The characterisation of vegetables has been a significant issue in the automatic picking with robotic arms in plant. Characterisation techniques commonly comprise destructive and non-destructive techniques, and non-destructive characterisation techniques are welcomed for the automatic detection in maturity degree and picking of robotic arms. Non-destructive techniques such as optical, ultrasonic, microwave, near infrared reflectance spectroscopy (NIRS), image and semiconductors have been successfully applied to the assessment of fruits and vegetables ${ }^{[3-12]}$. To detect unripe tomatoes, a sensor for predicting tomato maturity was developed based on spectral and colour features of green mature tomatoes ${ }^{[13]}$. Traditionally, fruits and vegetables were

Received date: 2018-09-19 Accepted date: 2019-04-22

Biographies: Yao Xu, under post-graduate, research interest: intelligent agricultural equipment, Email: 452378268@qq.com; Wenjing Zhu, PhD, Associate Professor, research interest: agricultural engineering, Email: zwj0410@foxmail.com; Xinhua Wei, PhD, Professor, research interest: intelligent agricultural equipment, Email: wei_xh@126.com; Hongwe Sun, Senior Engineer, research interest: mechanical design. Email: 450645390@qq.com;

*Corresponding author: Jinyang Li, PhD, Associate Professor, research interest: biological information sensing. Jiangsu University, Zhenjiang 212013 , China. Tel/Fax: +86-511-88797338, Email: by0817136@163.com. harvested prior to full maturity to avert the rot and decay during the period from harvest to consumption. In fact, respiration dominated and photosynthesis terminated after fruits and vegetables were plucked from plants. Thus the quality (such as flavour) of advanced picking fruits and vegetables are greatly reduced compared with naturally ripe ones. The quality of the tomatoes also depends on the maturity stage obtained at harvest ${ }^{[14]}$. Tomato is a very common and useful vegetable all over the world. Ripening tomatoes, which are rich in vitamins and minerals, are important to human health ${ }^{[15]}$. Hence, studying of tomato ripening is of great importance, which will not only help people to have better quality tomato but also help the researchers and scientists to analyse its taste, nutrient contents and quality.

Although the chemical and biological methods can be used to evaluate the ripening state and the quality of fruits, these techniques are destructive, time-consuming and hardly suitable for routine applications ${ }^{[16]}$. Besides, chemical and biochemical methods need to prepare special samples, special laboratory set up, expertise and time ${ }^{[16]}$. Therefore, to study the tomato ripening state and to evaluate the tomato nutrients levels noninvasively, a non-destructive tomato ripening process technique is essential for fast, reliable and repetitive monitoring. NIRS and computer vision have been applied in maturity evaluation. However, the confronting main problem of NIRS is the robustness of prediction model because the prediction performance of NIRS model varied with external factors such as temperature, light, water availability and production years ${ }^{[7]}$. To improve model robustness, a large amount of data at different production conditions which is difficult to obtained at most situations is necessary. Moreover, the sensitivity of computer vision to environmental factors such as light limited its practical use.

As a low cost and simple technique, electrical impedance spectroscopy (EIS) has been widely used to non-destructively 
characterise fruits and vegetables. Compared to other new microscopic techniques, the EIS is considered competitive because of the advantage in vivo detection of early changes both in physiological and structure stress ${ }^{[17,18]}$. It has been recently applied in the characterisation of fruits and vegetables in a very high frequency range ${ }^{[6,14,18-22]}$. At present, the assessments using EIS on maturity of apple ${ }^{[23]}$, mango ${ }^{[14]}$, durian ${ }^{[19]}$, banana ${ }^{[2]}$ and nectarine ${ }^{[3,4]}$ have been reported. These studies focus on the post-harvest fruits and the electrical impedance measurements were conducted off-line. However, few studies on the ripening evaluation of tomatoes based on EIS have yet reported. The changes in sugars, acids, $\mathrm{pH}$ and other quality parameters during extended vine holding of ripe processing tomatoes were studied, results indicated that vine holding of ripe fruit detrimentally impacts quality, especially $\mathrm{pH}$ and titratable acidity ${ }^{[24]}$. The intractable problem of high $\mathrm{pH}$ in tomato juice encountered by tomato processors was caused by increased average maturity at plucking ${ }^{[24]}$. $\mathrm{pH}$ is one of the important quality attributes of processing tomatoes. It has been advised that 4.4 is the maximum desirable $\mathrm{pH}$ value for food safety and the optimum target $\mathrm{pH}$ should be $4.25^{[25]}$. The electrical impedance of tomatoes was measured to investigate the physiology of fruit maturity; results show that the angular frequency for different samples such as cutin and cuticule of mature and immature fruits has different dielectric behaviour for each membrane ${ }^{[26]}$. However, the aim of these studies was also focused on the postharvest fruits and vegetables, hardly aim at the tree-ripening or plant-ripening fruits and vegetables.

A plenty of important physiological changes emerging in the fruits was associated with many variations such as ethylene production, respiration and enzyme activity ${ }^{[27]}$. In the post harvested state, as the fruits are removed from their source of water, minerals and sustenance, the fruits tissues continue to respire using available and stored sugars and organic acids, and they begin to senesce rapidly. In fact, photosynthesis terminated and respiration dominated after fruits and vegetables were detached from plants. It is suspected that the physiological property is different between postharvest fruits and plant-ripening fruits. The aim of this study is to discriminate the ripe status of plant-ripening tomatoes on a plant by measuring the impedance of tomatoes and attempt to determine the appropriate picking time.

\section{Materials and methods}

Experiments were conducted in the Venlo greenhouse of Jiangsu University, China $\left(32^{\circ} 11^{\prime} \mathrm{N}, 119^{\circ} 27\right.$ E), under controlled conditions such as photoperiod of $16 / 8 \mathrm{~h}$ (light/darkness), daily air temperature and relative humidity ranging from $21^{\circ} \mathrm{C}$ to $25^{\circ} \mathrm{C}$ and between $70 \%$ and $85 \%$, respectively, and light radiations of $800 \mu \mathrm{mol} /\left(\mathrm{m}^{2} \cdot \mathrm{s}\right)$. Experiments were carried out from April to August, 2016.

\subsection{Sample preparation}

Tomato seedlings (Hybrid "908") were cultivated with perlite and watered with nutrient solution in a Venlo greenhouse. Tomato fruits go through three different stages: green, white green and red. The branch with at least 4 tomato fruits was selected. After fruits turn white green, 60 tomatoes with uniform size, regular shape, without damage or defects were adopted in this study. Impedance measurement was conducted four times and the corresponding treatments were successively named as T1, T2, T3 and $\mathrm{T} 4$ along with progression of ripening process. The intervals of each measurement were $5 \mathrm{~d}$. Tap water was used to remove foreign material on the fruit surface while the impedance tested area was carefully cleaned using a tissue before impedance measurement. Volume and temperature are correctly recorded. To avoid injuring the fruit tissue and ensuring good contact between samples and electrodes, the proper contact force $(4 \mathrm{~N})$ between the electrodes and fruit skin are determined based on multiple tests .

\subsection{Volume measurement}

Considering the mass of plant-fruit was difficult to measure, the fruit volume was measured to overcome the effect of fruit size on impedance. Thus the bulk impedance was calculated and used in this study. The volume range of the measured tomato fruit is $196 \pm 17 \mathrm{~mL}$. The bulk impedance (BI) could be describes as: $B I=$ $\left(Z_{i}^{*} V_{i}\right) / V_{1}$, where $Z_{i}$ is the impedance at $i^{\text {th }}$ measurement. $V_{i}$ and $V_{1}$ were the volumes at the $i^{\text {th }}$ and first measurements, respectively. Because it was difficult to directly measure volume using conventional instrument for object with irregular shape, the "drainage method", i.e., the volume of water drained for the object was adopted in this study. The detailed steps were as follows. First, the tomato was completely immersed into a $500 \mathrm{~mL}$-break filled with water and then the spilled water was collected and the volume was recorded by measuring cylinder. The volume must be measured gently to avoid fruit being detached from plants.

\subsection{Soluble solid content determination}

Soluble solid content (SSC) are used as a measure of sugar content and are determined from detection on tomato fruit juice. Fruit juice was extracted for each tomato immediately after the impedance measurement. The sugar level of fruit increases from the calyx-end to the stem-end as is well-known. Therefore, tomatoes were cut into longitudinal slices in order to eliminate sugar discrepancy in different part of fruit. Then juice was extracted by putting tomato slices into a blender, centrifuged at $3000 \mathrm{r} / \mathrm{min}$ for $3 \mathrm{~min}$, and filtered through filter paper to get clear juice. The $\mathrm{pH}$ determination was conducted at a constant temperature of $25^{\circ} \mathrm{C}$ considering influence of temperature variation on $\mathrm{pH}$ measurement.

The SSC was determined by means of an Atago Pallete Series Model PR101 a digital refractometer (Atago Co. Ltd., Tokyo, Japan). The refractometer readings are known as Brix scale, which are regular expressed in percent total soluble solids by weight ${ }^{[28]}$. ${ }^{\circ}$ Brix was determined by a hand held refractometer with measuring range from 0 to $32 \%$ Brix. Data were read from the graduations by placing several drops of tomato fruit juice on the top of the prism assembly.

\section{4 pH determination}

The $\mathrm{pH}$ value of tomato juice was determined with a PHSJ-3F $\mathrm{pH}$ Meter (Shanghai Precise Scientific Instrument Co., Ltd, Shanghai, China). Three replicated $\mathrm{pH}$ readings were averaged for each sample.

\subsection{Bulk impedance measuring system}

Considering the fruits such as apple and tomato with regular shape, impedance values keep nearly constant value when measured was conducted across a definite periphery which possessing approximately same diameter ${ }^{[14]}$. The three pairs of measurement points on the circumference, $120^{\circ}$ apart, were selected and the three corresponding readings were obtained for each tomato. The measurement values were calculated for three replicated measurements on each sample.

The vivo impedance measurements of fruits were performed with a combination of Solartron 1260A Impedance/Gain-Phase Analyzer and 1294 impedance interface (Solartron Analytical, 
England, UK). It extends the measurement capability of the frequency response analyser to address such applications as bio-impedance spectroscopy and high impedance materials testing. The data from impedance analyser were automatically transmitted to a personal computer (PC) through a GPIB interface. The measurement system is shown in Figure 1 and the experimental device can be seen in Figure 2.

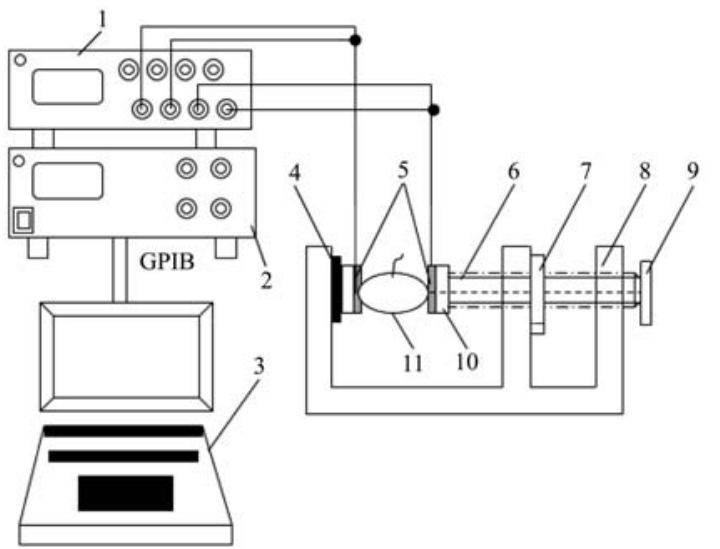

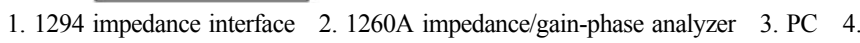
Pressure sensor 5. Electrodes 6. Screw 7. Nut 8. Support frame 9. Regulating handle 10. Insulation pad 11. Tomato fruit.

Figure 1 Measurement system of tomato fruits via two electrodes.

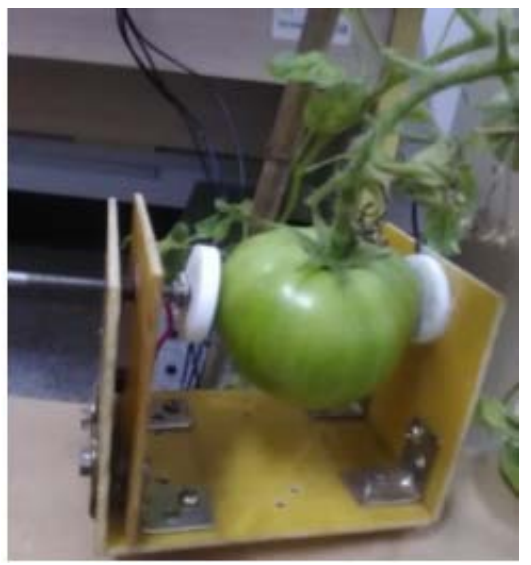

Figure 2 Experimental device for electrical impedance measurement of tomato fruit

Measuring electrodes included pressure sensor, two copper electrodes, insulation pad, screw, nut, support frame and regulating handle. Two electrodes were glued to insulation pads with insulation glue. EIS is based on the measurement of current that passed through the samples. Electric current was closely associated with the contact area between the sample and electrode. The impedance value was influenced by contact area between electrodes and fruit ${ }^{[14]}$. The large contact area will lead to lower resistance and higher capacitance and hardly ensure the good contact with the fruit where predominately have curved surfaces. The small cross-section area will cause higher impedance and be able to keep good contact with the fruit surface. Various cross-sectional areas were tested and copper electrodes with $10 \mathrm{~mm}$ diameter ( $1 \mathrm{~mm}$ thickness) were finally adopted in this study. Fruits with maximum height of $6 \mathrm{~cm}$ and width of $8 \mathrm{~cm}$ were suitable for test by the designed device. The higher potential electrode is fixed to the one terminal of screw and the nut is fixed on the support frame. The pressure sensor is pasted on the support frame and the lower potential electrode is pasted on the top surface of pressure sensor. The lower potential electrode kept stationary and the contact force between electrodes and measured objects was controlled by adjusting the nut-bolt mechanism. Considering the mass of plant-fruit was difficult to measure online, the fruit volume was measured to overcome the effect of fruit size on impedance. Thus the bulk impedance was calculated by dividing fruit impedance by volume and used in this study. To explore the effect of temperature on impedance, temperature measuring system was designed based on AD590, microcontroller AT89S52 and liquid crystal display 12864. The pressure sensor is a piezoresistive sensor FSR402 (interlink electronics, Co., Ltd., USA) with $0-100 \mathrm{~N}$ measurement range and $12.7 \mathrm{~mm}$ diameter effective detection area. The output resistance of sensor rise with the increase of force applied to the sensor surface, and thus the pressure force of electrode on fruit is turned into resistance $\left(R_{F}\right)$. To facilitate sensing the force signal, the output resistance of sensor was turned into corresponding voltage signal. The detailed circuit presents in Figure 3. ME7660 is a charge pump DC-DC voltage converter used for converting the $+5 \mathrm{~V}$ into $-5 \mathrm{~V}$ and providing reference voltage for sensor FSR402. LM324 is a high-performance operational amplifier used for amplifying the output voltage of FSR402 into $U_{0}$. This voltage $U_{0}$ is converted by an $\mathrm{A} / \mathrm{D}$ and the corresponding contact force is calculated.

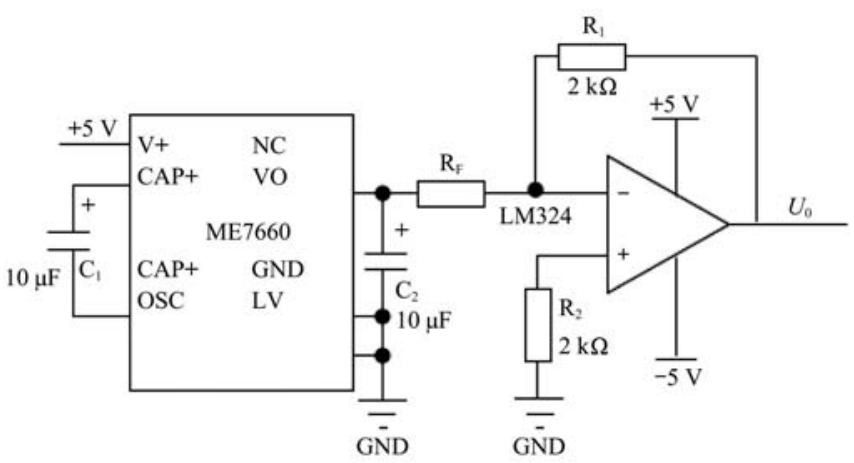

Figure 3 Pressure detection circuit

The two-terminal and four-terminal structures are commonly adopted measured patterns. Despite four-terminal method offer the advantage of reducing electrode polarization impedance $(E P I)^{[22]}$, two-electrode measurement method was used in this study considering the convenience in clipping the tomato fruit. EPI can cause inaccuracies in the measurement at low frequencies ${ }^{[3]}$. The EPI is mainly apparent at frequencies below $20 \mathrm{~Hz}^{[29]}$. The measured values under frequencies above $1 \mathrm{kHz}$ were analysed in this study to decrease EPI. To avoid self-heating and injuring plants and thus influencing subsequent normal growth, impedance measurements were measured at $0.1 \mathrm{~V}$ and scanned 91 spot frequencies (logarithmic frequency intervals) between $1 \mathrm{~Hz}$ and 1 MHz. The contact points between electrode and tomato tissue was symmetrically along the radial direction as shown in Figure 2. Moreover, to avoid the contamination of the juice from the first test samples to subsequent test samples and juice corrosion to metal electrodes, electrodes were washed with deionised water and wiped dry with a filter paper after each measurement. Any contact between leaves and fruits should be averted to improve the accuracy of impedance measurements.

\section{Results and discussion}

\subsection{Effect of maturity on $\mathrm{pH}$ and soluble solids content of tomato fruit}

$\mathrm{pH}$ is a significant quality index of processing tomatoes and SSC is a frequently-used measure for assessing tomato quality ${ }^{[24]}$. The $\mathrm{pH}$ and SSC of tomato gradually rise as the increase of 
maturity (Figure 4 and Figure 5). This is consistent with what has been reported previously ${ }^{[24]}$. The $\mathrm{pH}$ range of tomato fruits is 4.0-4.4. The sharp increase in $\mathrm{pH}$ and SSC occurs during the period from $\mathrm{T} 2$ to $\mathrm{T} 3$ while corresponding changes are slow at the other two stages from $\mathrm{T} 1$ to $\mathrm{T} 2$ as well as from $\mathrm{T} 3$ to $\mathrm{T} 4$. This may because that the stage from $\mathrm{T} 2$ to $\mathrm{T} 3$ has sharp jump in respiration and production of ethylene that accelerates the maturity. Furthermore, respiration action gradually dominated as fruits ripening. Higher respiratory leads to strong utilization of acids, during which the main acids such as citric and malic acids in fruits were oxides and ATPs were generated for synthesis of new compounds $^{[30]}$.

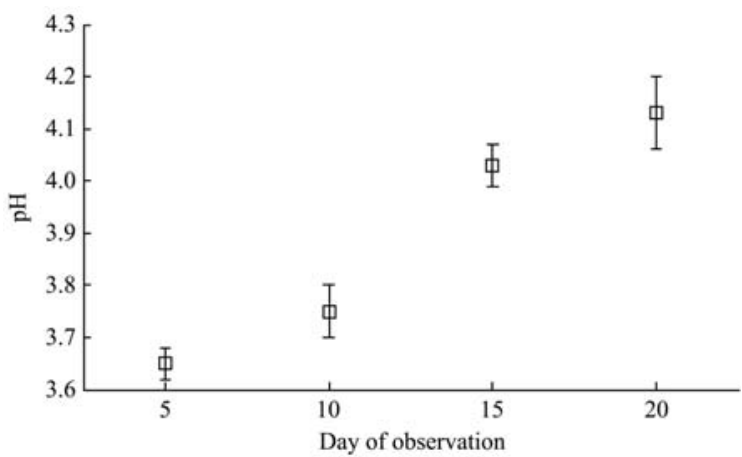

Figure 4 Variation in fruit $\mathrm{pH}$ with respect to ripening degree.

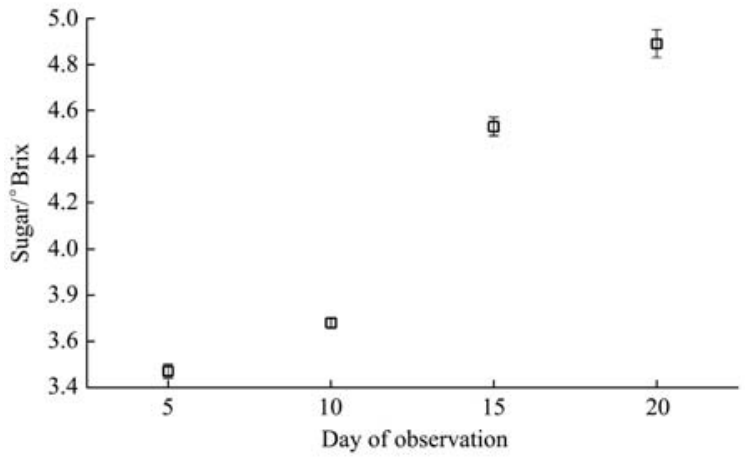

Figure 5 Variation in fruit SSC with respect to ripening degree

\subsection{Determination of optimal EIS parameters}

Variation in impedance, phase angle and capacitance with frequency and the reactance against resistance were analysed to determine the frequencies where an obvious difference in the EIS parameters was found with respect to the change in ripening stage. The curve fitting was performed by Z-view software, and Matlab 7.1 software was used to conduct data analysis.

\subsubsection{Change in impedance with respect to frequency}

EIS studies carried out during tomato plant-ripening showed that the impedance (Figure 6a), capacitance (Figure 6c), phase angle (Figure 6d), resistance (Figure 6e), reactance (Figure 6f) and Cole-Cole arc (Figure 6b) of tomato varied with the progress of ripening as well as with the frequency. The impedance gradually increases as the tomato gets ripened in a range of measured frequencies. The result coincided with the study for banana by Atanu et al. ${ }^{[2]}$ The reason is probably attribute to the increase in sugar content and other subsequent physiological changes such as decrease in acidic or salt. Moreover, the deduction of iron parts in tomato tissue may also causes decline of tissue conductivity and thus increases impedance. The conductance of an aqueous solution is closely correlated with the ion content of the samples. Moreover, impedance measurements also depended on the ions capability of movement under the action of an electrical field in this aqueous solution ${ }^{[31]}$. However, the impedance value was persistently declining along with the increase of the durian maturity ${ }^{[19]}$. Their result was contradicted with the result in this study, which can be explained that the number of cortical sclereid in the outer part of the lower stem increased as the durian matured and the increase in sclereid lead to the more electrolytes in the extracellular liquid and decrease in impedance of durian ${ }^{[19]}$.

For biological tissue, cell can be expressed as cell membrane, intracellular and extracellular fluids which are separated by cell membrane. The cell membrane can be represented by a capacity and a resistor in parallel with two additional series resistors characterised intracellular and extracellular fluids ${ }^{[32]}$. The impedance of biological tissue drops with the increase in frequency due to the capacity characteristics of the cell membrane.

3.2.2 Change in capacitance with respect to frequency

A sharp decrease in capacitance (C) was observed up to around $10 \mathrm{kHz}$ and then the decrease rate was slow and started to level off towards high frequency region (Figure 6c). This phenomenon was in agreement with the results by Rehman et al. ${ }^{[33]}$ for mangos and by Pramote et al. ${ }^{[19]}$ for durians. A monotonous drop in $\mathrm{C}$ value was visually observed as tomato fruits matured. The membrane permeability of tomatoes increased as the progression of ripening process ${ }^{[27]}$. Therefore, it is speculated that a decrease in $\mathrm{C}$ was caused by the increase of membrane permeability. The distinction in $\mathrm{C}$ value is obvious at $1 \mathrm{kHz}$.

\subsubsection{Change in reactance with respect to resistance}

Resistance and the absolute value of reactance were plotted in the Nyquist plot from $100 \mathrm{~Hz}$ to $1 \mathrm{MHz}$ with respect to maturity ( Figure 6b). The curve displays a semielliptical arc with its centre below the X-axis. The curve can be described by the modified model shown in Figure 7. A monotonous decrease in the resistance was visually observed as the increased frequency (from right to left in Figure 6b). The absolute value of reactance of samples with different maturity reached to a peak near $1 \mathrm{kHz}$ and descended as the frequency continuously increased. The frequency of $1 \mathrm{kHz}$ was afterwards defined as the peak frequency where maximum reactance value occurred.

At high frequency region (the left side of Figure $6 \mathrm{~b}$ ), the Cole-Cole plot was close while the obvious divergence in plot for sample with different maturity was observed at low frequencies. As the fruits ripened, the semielliptical arc continuously expanded, which was contrary to the ripening of nectarine fruit ${ }^{[4]}$ and the loss of moisture content in sliced cucumber ${ }^{[22]}$. The capacitance of biological membranes was normally quantified by the reactance of biological tissue ${ }^{[4]}$. As the fruits matured, the membrane permeability gradually increased and thus resulted in the drop in C. Therefore, it is speculated that the semielliptical arc extended as ripening was caused by the drop in $\mathrm{C}$. As such, the reactance exhibited divergence in low frequencies over the course of maturity.

The Cole-Cole plots from samples with different maturity were close at high frequencies. This phenomenon can be explained that at high frequencies, the capacity of cell membrane $C_{m}$ can be ignored because the value of $C_{m}$ is far less than that of other parameters. Thus, impedance mainly depends on extracellular and intracellular resistances. Though ripening degree had an evident effect on cell membrane $C_{m}$, this can be ignored at high frequencies, and thus measurement values from different treatments are close. Moreover, the polarization in the material requires sufficient time. The high frequency signifies a short polarization time, which resulted in complete polarization. As a result, the total polarization was decreased by the increased frequency. 
3.2.4 Change in phase angle of impedance with respect to frequency

The phase angle $(\theta)$ continuously drop in value up to peak value and then rise as the increased frequency (Figure 6d).

In the low-frequency region, in view of the high electric capacity of cell membranes, the electrical current mainly flows through extracellular fluid, which presents relatively high resistance and dominates in total impedance ${ }^{[34]}$. Thus $\theta$ value is relatively small. With the increase of frequency, the current flow through the intracellular and extracellular fluids. The proportion of reactance of the membranes in total impedance gradually increases and thus $\theta$ increases continuously. At high frequencies, the total impedance mainly depends on the intracellular and extracellular resistances because the reactance actioned by membrane capacity gradually decreases. As a result, the value in $\theta$ continuously reduces.

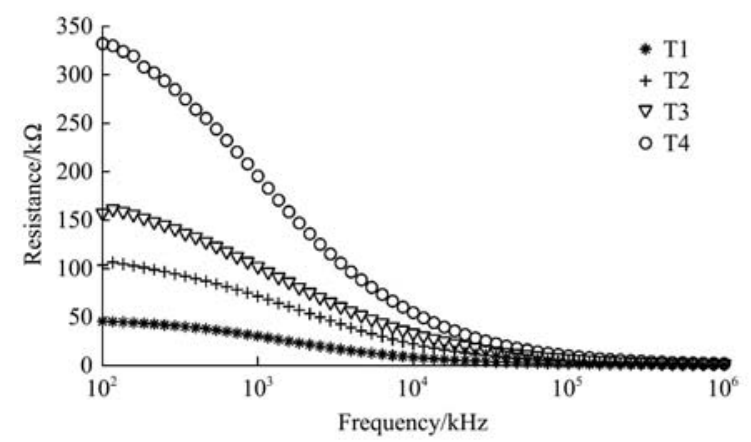

a. Impedance vs. frequency characteristics of tomato

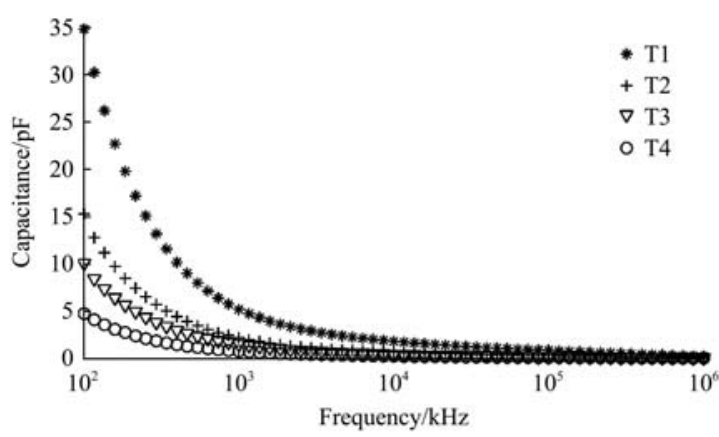

c. Capacitance vs. frequency characteristics of tomato

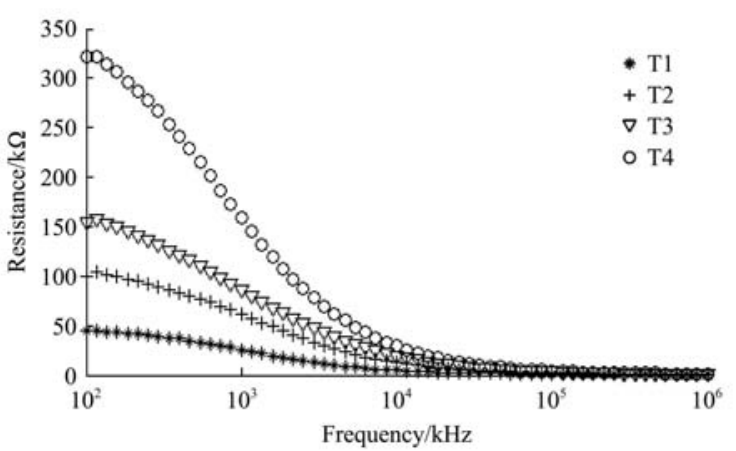

e. Resistance vs. frequency characteristics of tomato
Moreover, the valley value in $\theta$ increased along with progression of ripening process. The capacitance reduces with maturity and thus the share of reactance in total impedance increases.

The lower tissue homogeneity (geometry and composition) implied the higher parameter dispersion and lower phase angle ${ }^{[35]}$. As fruit ripening, the fruit tissue became uniform because the composition changes from the combination of solid and liquid phase to the tissue dominated by liquid phase composition. As a consequence, the phase angle offered high values with the progression of maturity.

The phase angle variation also indicated that the characteristic frequency at the maximum phase angle appears different and increased as maturity. This is due to the drop in the reactance in the tomato during the process. Coincides with the results by Atanu et $\mathrm{a}^{[2]}$ for banana.

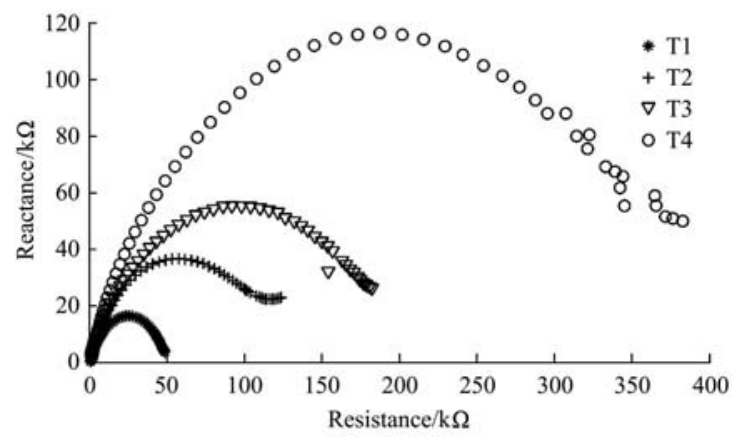

b. Cole-Cole arc variations for tomato

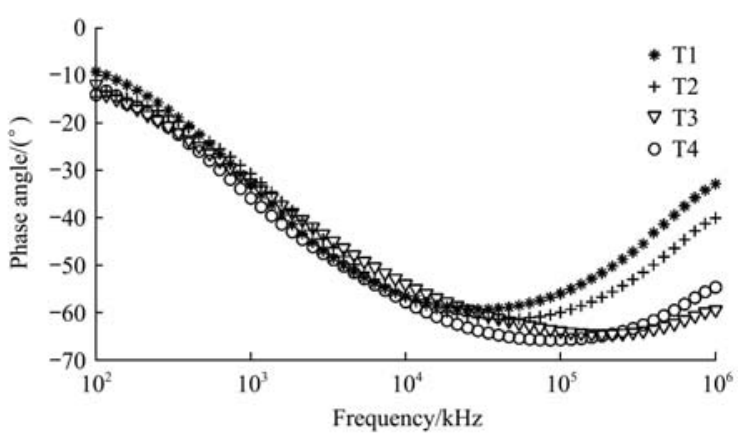

d. Phase angle vs. frequency characteristics of tomato

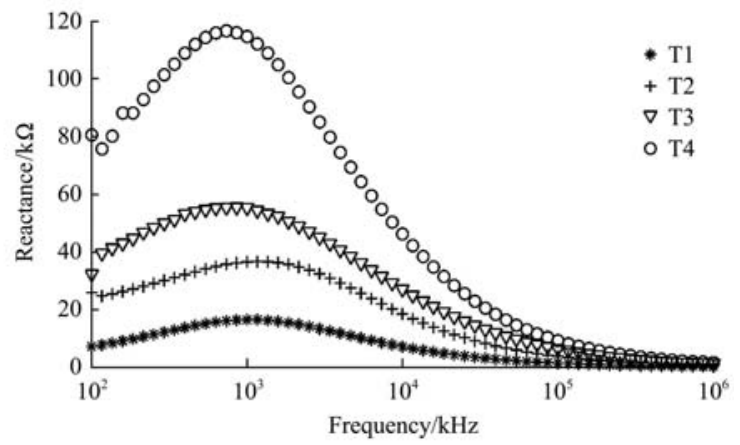

f. Reactance vs. frequency characteristics of tomato

Figure 6 Variation in the impedance spectra of tomato fruit for different ripening stage

\subsection{Development of equivalent circuit model}

The physical properties of the materials can be quantified by monitoring the changes of equivalent circuit parameters.

The equivalent circuit model is developed with Z-view software, and the Nyquist plots are fitted. Based on Nyquist curve fitting, the equivalent circuit parameters about the tomato fruits are acquired. Equivalent parameters were estimated using complex nonlinear least square (CNLS) curve-fitting ${ }^{[36]}$. For the CNLS fitting, the basic rule must be observed is that the sum of the squares of real and imaginary residuals was minimized. During the development of the equivalent circuit model, several different models were tested to acquire model with best performance. By analyzing the fit results, it is found that the equivalent circuit model as shown in Figure 7 presents the optimum fit model and good results with Chi-squared 0.006833 and sum of sqr 0.97032 , and this model was selected for subsequent analysis. 
To describe the variations in the impedance characteristics shown in Figure 6 quantificationally, equivalent circuit analysis was conducted. To test the validity of model fitting, samples without involving model development and fitted the established equivalent circuit model to experimental impedance values were randomly selected to evaluate the fitting accuracies. Figure 8 shows the comparison between the approximation results of the models and experimental results of tomato fruit $(10 \mathrm{~d}$ after fruit begin to turn white). It can be seen that the measured values for $Z$, $R, X$ and $\theta$ with corresponding $R_{R}^{2}, R_{X}^{2}$, and SD of $0.987,0.975$, and $43 \Omega$, respectively. This fact demonstrates that the developed model as show in Figure 7 presents good performance and can be used to evaluate the maturity of tomato fruit. $R_{R}^{2}, R_{X}^{2}$, and SD can be calculated by the method found in $\operatorname{Re}^{[37,38]}$. Considering $Z$ and $\theta$ can be described by $R$ and $X$, that is, $R=|Z| \cos \theta, X=|Z| \sin \theta$, the fitting accuracy for $R$ and $X$ imply those of $Z$ and $\theta$.

The model (in Figure 7) was used to analyze the impedance spectra of samples with different ripening degree. The experimental data (measurement value) presents a perfect fit with the approximations (calculation values). The values of $R_{R}^{2}$ for

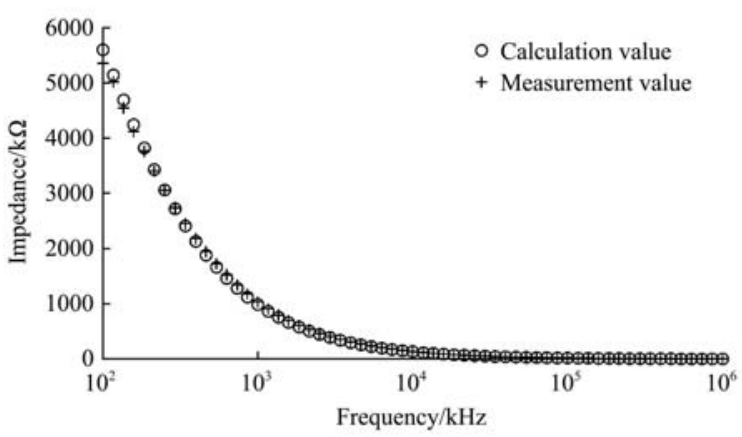

a
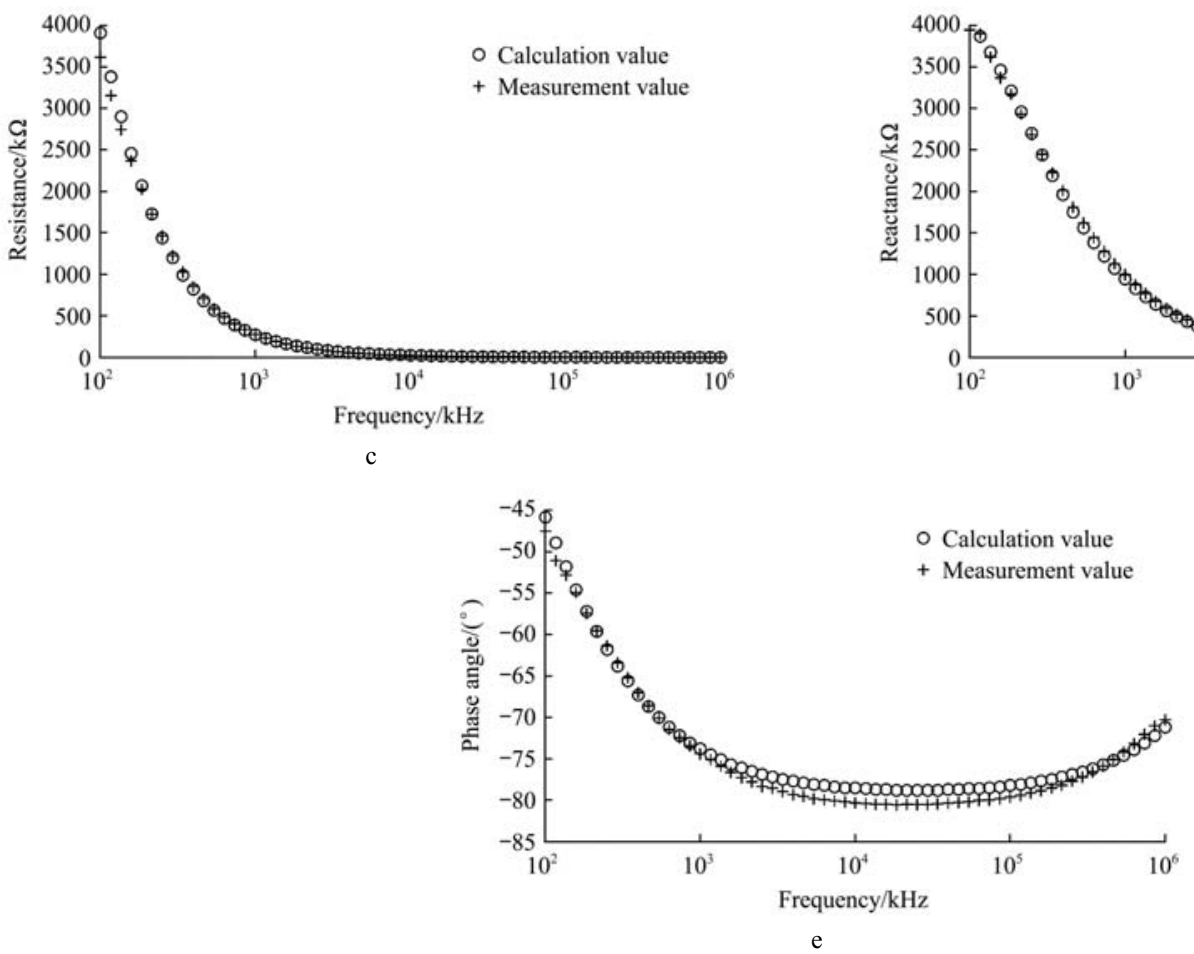

Figure 8 Curve fitting in Z-view each sample were between 0.936 and 0.989 , and the values of $R_{X}^{2}$ were between 0.941 and 0.993 . The ratio of the standard deviation and average values (variation coefficient) were less than 0.869 for measured $R, 0.902$ for calculated $R, 0.953$ for measured $X$, and 1.072 for calculated $X$, respectively. From above analysis, the developed equivalent circuit model is highly efficient for evaluating the ripening of tomato fruit.

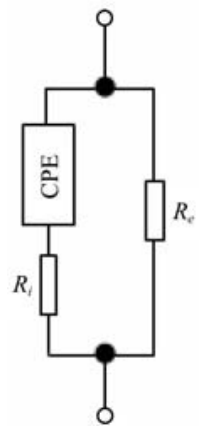

Note: CPE: constant phase element; $R_{i}$ : intracellular resistance, and $R_{e}$ : extracellular resistance.

Figure 7 Equivalent circuit model for tomato during ripening

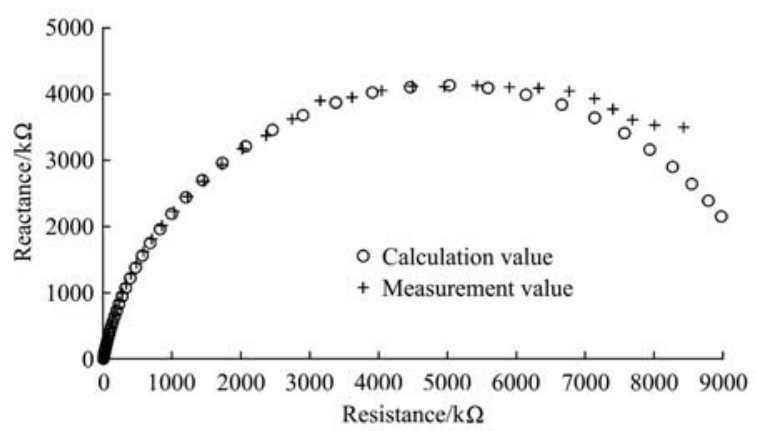

$\mathrm{b}$

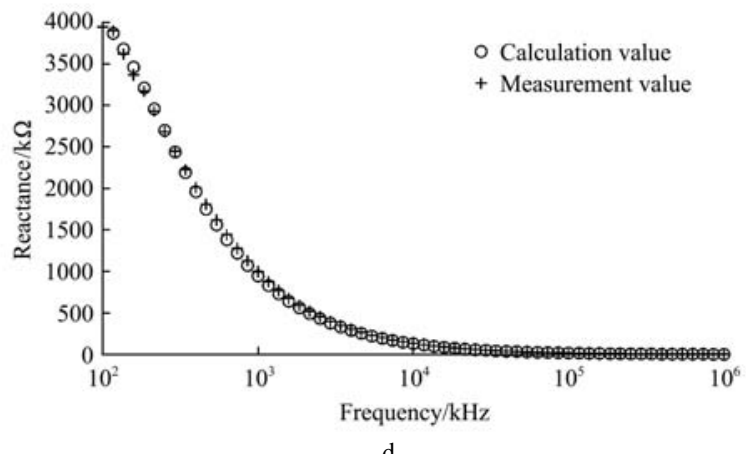

d
To examine the impedance variation of different samples, the impedance responses from different samples from same branch were studied. Three different tomato samples (named as S1, S2 and S3) from a same branch were selected and the impedance responses were analysed using EIS (Figure 9). It is observed that the change of impedance parameters is low for the samples from a same branch. Cole-Cole plots of the three tested samples are visually close to each other. This results shows that the ripening 
stage of all other samples can be predicted and assessed by the impedance spectroscopy from one sample.

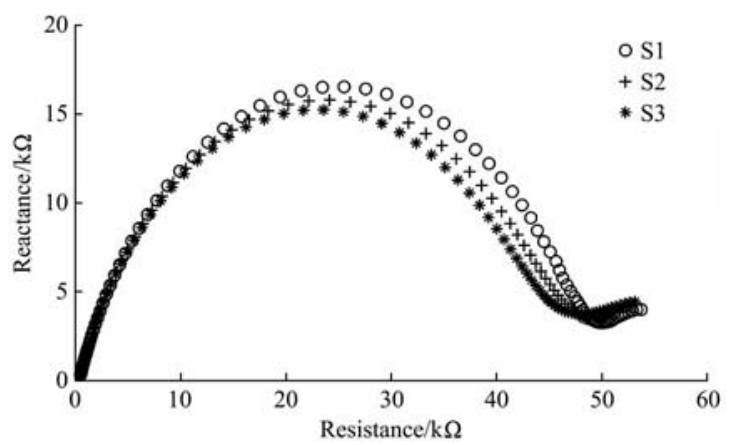

Figure 9 Impedance variation in different samples from a same tomato branch

\subsection{Maturity classification of tomato}

The size difference of impedance arc at different ripening stages implied that the arc size can be used as one of the prediction variables. The arc size can be characterised by the five parameters including $R_{\max }$ ( $R$ value at maximum frequency of $1 \mathrm{MHz}), R_{\min }\left(R\right.$ value at minimum frequency of $100 \mathrm{~Hz}$ ), as $X_{\max }$ ( $X$ value at maximum frequency of $1 \mathrm{MHz}), X_{\min }(X$ value at minimum frequency of $100 \mathrm{~Hz})$ and $X_{\text {peak }}(X$ value at peak frequency of $1 \mathrm{kHz}$ where the $X$ value is maximal). Therefore, the optimal frequencies were selected as $100 \mathrm{~Hz}, 1 \mathrm{kHz}$ and $1 \mathrm{MHz}$.

To test the performance of ripening classification using EIS parameters, the further investigation was performed. In order to distinguish mature tomato, analysis was conducted using the five EIS parameters mentioned above $\left(R_{\max }, R_{\min }, X_{\max }, X_{\min }\right.$ and $\left.X_{\text {peak }}\right)$. The results (Table 1) showed that the classified model presented good performance with an accuracy of $86.7 \%$.

Table 1 Discriminate results showing performance of classification into two maturity class based on five electrical impedance parameters

\begin{tabular}{ccccccc}
\hline \multirow{2}{*}{$\begin{array}{c}\text { Electrical impedance } \\
\text { parameters }\end{array}$} & $\begin{array}{c}\text { Actual } \\
\text { group }\end{array}$ & $\begin{array}{c}\text { Correct } \\
\text { Rate } \%\end{array}$ & \multicolumn{3}{c}{ Predicted group } \\
\cline { 4 - 6 } & & & Immature & mature & Total \\
\hline \multirow{2}{*}{$Z_{\text {max }}, Z_{\min }, X_{p k}, \theta_{p k}$} & mature & 89.5 & 4 & 34 & 38 \\
& Total & 88.3 & 23 & 37 & 60 \\
\hline
\end{tabular}

\section{Conclusions}

Tomato plant ripening has been analysed non-destructively using EIS and the electrical impedance variations during the ripening was investigated. The results showed that the impedance, phase angle, resistance, reactance and capacitance increased with the progression of maturity. The Cole-Cole plots gradually enlarged during ripening. The equivalent circuit including CPE were acquired by CNLS fitting with high accuracy. The obtained classified model by discriminate analysis presented good performance with an accuracy of $88.3 \%$. The sensitive frequencies $100 \mathrm{~Hz}, 1 \mathrm{kHz}$ and $1 \mathrm{MHz}$ for discriminating tomato ripening were selected and the five corresponding sensitive electrical parameters were determined. Impedance analysis for different samples from the same branch implied that the ripening stage of all other samples can be predicted and assessed by the impedance spectroscopy from one sample. Therefore, the method conducted on limited samples can be extended to characterise the ripening status of entire tomato samples. This study laid the foundation for determining the appropriate picking time by robotic arms. However, the mathematical model perhaps varies for different tomato variety because of the difference in physiology and microstructure. Therefore, a systematic and comprehensive study for different varieties is necessary in future to extend the application scope. Moreover, in order to improve the accuracy, the quantitative relationships between the electrical impedance parameters and physiological parameters such as $\mathrm{pH}$, soluble solids content and titratable acid, etc. should be developed.

Although two-terminal configuration was applied in the frequency range from $100 \mathrm{~Hz}$ to $1 \mathrm{MHz}$ to minimise the EPI, the contact resistance which led to error was still remained. Four-terminal configuration which is an efficient technique to decrease polarization impedance can be adopted in the future investigation. Besides, the effect of temperature on impedance during measurement should be considered.

\section{Acknowledgements}

The authors gratefully acknowledge the financial support from Natural Science Foundation of Jiangsu Province (BK20161346), Natural Science Youth Fund of Jiangsu Province (BK20150493) and Priority Academic Program Development of Jiangsu Higher Education Institutions (Jiangsu fiscal education 2014-37).

\section{Nomenclatures}

$\begin{array}{cl}\text { BI } & \text { bulk impedance } \\ \text { C } & \text { capacitance }(\mathrm{pF}) \\ \text { CNLS } & \text { complex nonlinear least square } \\ \text { CPE } & \text { constant phase element } \\ \text { EIS } & \text { electrical impedance spectroscopy } \\ \text { NIRS } & \text { near infrared reflectance spectroscopy } \\ \text { R } & \text { resistance }(\Omega) \\ \text { RMSE } & \text { root-mean-square error } \\ \text { SD } & \text { standard deviation } \\ \text { X } & \text { Reactance }(\Omega) \\ Z & \text { impedance } \\ |Z| & \text { impedance magnitude }(\Omega) \\ \theta & \text { phase angle of impedance }\end{array}$

\section{[References]}

[1] Kader A A. Fruit maturity, ripening, and quality relationships. In International Symposium Effect of Pre- and Postharvest Factors on Storage of Fruit. Acta Horic, 1999; 485: 203-208.

[2] Atanu C, Tushar K B, Dibyendu G, Badal C. Electrical impedance variations in banana ripening: an analytical study with electrical impedance spectroscopy. Journal of Food Process Engineering, 2017; 40(2): 1-14.

[3] Harker F R, Dunlop J. Electrical impedance studies of nectarines during cool storage and fruit ripening. Postharvest Biology and Technology, 1994; 4(1): 125-134.

[4] Harker F R, Maindonald J H. Ripening of nectarine fruit. Plant Physiology, 1994; 106:165-171.

[5] Inaba A, Iwamoto T. Electrical impedance analysis of tissue properties associated with ethylene induction by electric currents in cucumber (Cucumis sativus L.) fruit. Plant Physiology, 1995; 107(1): 199-205.

[6] Bauchot A D, Harker R F, Michael A W. The use of electrical impedance spectroscopy to assess the physiological condition of kiwifruit. Postharvest Biology and Technology, 2000; 18: 9-18.

[7] Thibault N, Jacques J, Fabrice D, Marc C, Mathieu L. Robust NIRS models for non-destructive prediction of mango internal quality. Scientia Horticultrae, 2017; 216: 51-57.

[8] Dolores P M, Maria T S, Patricia P, Maria A S, Jose E G, Ana G V. Non-destructive determination of quality parameters in nectarines during on-tree ripening and post-harvest storage. Postharvest Biology and Technology, 2009; 52: 180-188. 
[9] Jha S N, Narsaiah K, Jaiswal P, Bhardwai R, Gupta M, Kumar R, Sharma R. Nondestructive prediction of maturity of mango using near infrared spectroscopy. J. Food Eng, 2014; 124: 152-157.

[10] Saranwong S, Sornsrivichai J, Kawano S. Prediction of ripe-stage eating quality of mango fruit from its harvest quality measured nondestructively by near infrared spectroscopy. Postharvest Biol, Tecnol, 2004; 31: 137-145

[11] Subedi P, WalshK B, Owens G. Prediction of mango eating quality at harvest using short-wave near infrared spectrometry. Postharvest Biol, Technol, 2007; 43: 326-324.

[12] Stefany C P, Jorge C P, Juan V M M, Georgina C D, Ruben L S, Maria J P F, Israel A V. Evaluation of the ripening stages of apple (Golden Delicious) by means of computer vision systems. Biosystems Engineering, 2017; 159: 46-58.

[13] Hahn F. Multi-spectral prediction of unripe tomatoes. Biosystems Engineering, 2002; 81: 147-155

[14] Rehman M, Basem A J A, Izneid A, Abdullan M Z, Arshad M R. Assessment of quality of fruits using impedance spectroscopy. International Journal of Food Science and Technology, 2011; 46: 1303-309.

[15] Padmanabhan P A, Paliyath. Solanaceous fruits including tomato, eggplant, and peppers. Encyclopedia of Food \& Health, 2016: 24-32.

[16] Anowar hossain M D, Rana M, Kimura Y, Roslan H A. Changes in biochemical characteristics and activities of ripening associated enzymes in mango fruit during the storage at different temperatures. BioMed Res. Int, 2014; 2014(2014): 1-11.

[17] Vozáry E, Jócsák I, Droppa M, Bóka K. Connection between structural changes and electrical parameters of pea root tissue under Anoxia: in P. Padilla (Eds.) Anoxia, Tech, Croatia, Rijeka, 2012; pp.131-146.

[18] Khaled D El, Castellano N N, Gazquez J A, García Salvador R M, Manzano-Agugliaro F. Cleaner quality control system using bioimpedance methods: A review for fruits and vegetables. Journal of Cleaner Production, 2015; 140: 1749-1762.

[19] Pramote K, Anupun T. Minimally-destructive evaluation of durian maturity based on electrical impedance measurement. Journal of Food Engineering, 2013; 116: 50-56.

[20] Tomkiewicz D, Piskier T. A plant based sensing method for nutrition stress monitoring. Precis. Agric, 2012; 13(3): 370-383.

[21] Muñoz-Huerta R F, ORTIZ-Melendez A D J, Guevara-Gonzalez R G, Torres-Pacheco I, Herrera-Ruiz G, Contreras-Medina L M, et al. An analysis of electrical impedance measurements applied for plant $\mathrm{N}$ status estimation in lettuce (Lactuca sativa). Sensors, 2014; 14: 11492-11503.

[22] Liu X. Electrical impedance spectroscopy applied in plant physiology studies. M.Sc. Thesis; RMIT University, Melbourne, Australia, 2006.

[23] Jackson P J, Harker F R. Apple bruise detection by electrical impedance measurement. Postharvest Biology and Technology. HortScience, 2000; 35(1): 104-107.

[24] Anthon G E, LeStrange M, Barrett D M. Changes in pH, acids, sugars and other quality parameters during extended vine holding of ripe processing tomatoes. J Sci Food Agric, 2011; 91(7): 1175-1181.

[25] Monti L M. The breeding of tomatoes for peeling. Acta Hortic, 1980; 100: 341-349.

[26] Benavente J, Ramos-Barrado J R, Heredia A. A study of the electrical behaviour of isolated tomato cuticular membranes and cutin by impedance spectroscopy measurements. Colloids and Surfaces A: Physicochemical and Engineering Aspects, 1998; 140: 333-338.

[27] Pangaribuan D H, Irving D E. The physiology and nutrition of tomato slices as affected by fruit maturity and storage temperature. Jurnal Agrista, 2006; 10(3): 142-151.

[28] Nelson S N. Frequency- and temperature-dependent permittivities of fresh fruits and vegetables from 0.01 to $1.8 \mathrm{GHz}$. Transaction of the ASAE, 2003; 46(2): 567-574.

[29] Ferris C D. Introduction to Bioelectrodes. Plenum Press, New York, 1974.

[30] Ladaniya M S. Citrus fruit, biology, technology and evaluation. Elsevier Inc, Printed and bound in the USA, 2008; 574p.

[31] Rafael M, Miguel A, Ana F, Franciny C S, José M B, Luis G, et al. Design of a low-cost non-destructive system for punctual measurements of salt levels in food products using impedance spectroscopy. Sensors and Actuators A, 2010; 158: 217-223.

[32] Angersbach A, Heinz V, Knorr D. Electrophysiological model of intact and processed plant tissues: cell disintegeration criteria. Biotechnology Progress, 1999; 15: 753-762.

[33] Rehman, M, Abu Izneid BAJA, Abdullah M Z, Arshad M R. Assessment of quality of fruits using impedance spectroscopy. International Journal of Food Science and Technology, 2011; 46(6): 1303-1309.

[34] Li X S, Xu G, Huang L. Effect of plant growth regulator on electrical impedance spectroscopy during ripening process in kiwifruits. Transaction of the CSAE, 2015; 31(1): 288-293. (in Chinese).

[35] Varlan A R, Sansen W. Nondestructive electrical impedance analysis in fruit: normal ripening and injuries characterization. Electro-Magnetobiology, 1996; 15: 213-227.

[36] Macdonald J R. Impedance spectroscopy. Annals of Biomedical Engineering, 1992; 20: 289-305.

[37] Li M Q, Li J Y, Mao H P, Wu Y Y. Diagnosis and detection of phosphorus nutrition level for Solanum lycopersium base on the electrical impedance spectroscopy. Biosystems Engineering, 2016; 143: 108-118.

[38] Li J Y, Li M Q, Mao H P, Zhu W J. Diagnosis of potassium nutrition level in Solanum lycopersicum based on electrical impedance. Biosystems Engineering, 2016; 147: 130-138. 